\title{
Autophagy inhibition enhances isobavachalcone-induced cell death in multiple myeloma cells
}

\author{
SHAN ZHAO ${ }^{1 *}$, CHUN-MIN MA $^{1 *}$, CHUAN-XU LIU $^{1}$, WEI WEI $^{2}$, YUN SUN $^{3}$, HUA YAN $^{2}$ and YING-LI WU ${ }^{1}$ \\ ${ }^{1}$ Department of Pathophysiology, Chemical Biology Division of Shanghai Universities E-Institutes, Key Laboratory of \\ Cell Differentiation and Apoptosis of National Ministry of Education; ${ }^{2}$ Department of Hematology, Rui-Jin Hospital, \\ Shanghai Jiao-Tong University School of Medicine; ${ }^{3}$ Department of Clinical Laboratory, \\ Dong-Nan Hospital, Shanghai, P.R. China
}

Received April 12, 2012; Accepted June 13, 2012

DOI: $10.3892 / \mathrm{ijmm} .2012 .1066$

\begin{abstract}
Despite recent advancements in therapeutic drugs, multiple myeloma remains an incurable disease. Therefore, a more effective treatment is urgently required. In this study, we show that isobavachalcone (IBC), a natural chalcone compound, induces apoptosis- and autophagy-related cell death in myeloma cells. The inhibition of autophagy by knocking down beclin-1 or by using autophagy inhibitors, such as 3-methyladenine, bafilomycin A and chloroquine significantly enhanced IBC-induced cell death, as demonstrated by the increased number of Annexin V-positive cells. Moreover, we demonstrate that the collapse of the mitochondrial membrane potential contributes to chloroquine and IBC-induced cell death, which is accompanied by the activation of caspase-9, and -3 , the cleavage of poly (ADP-ribose) polymerase (PARP) and the proteolytic activation of protein kinase $\mathrm{C} \delta$ (PKC $\delta$ ). Furthermore, the inhibition of the activation of PKC $\delta$ by rottlerin, an inhibitor of PKC $\delta$, not only suppressed the activation of PKC $\delta$, but also the apoptosis induced by the co-treatment of chloroquine and IBC, indicating the involvement of PKC $\delta$ in chloroquine plus IBC-induced cell death. Finally, the combination of chloroquine and IBC had little effect on the viability of normal peripheral blood mononuclear cells. As both chloroquine and IBC have been shown to be
\end{abstract}

Correspondence to: Professor Ying-Li Wu, Department of Pathophysiology, Chemical Biology Division of Shanghai Universities E-Institutes, Key Laboratory of Cell Differentiation and Apoptosis of National Ministry of Education, Shanghai Jiao Tong University School of Medicine, No. 280 Chongqing South Road, Shanghai 200025, P.R. China

E-mail: wuyingliwu@163.com

Dr Hua Yan, Department of Hematology, Rui-Jin Hospital, Shanghai Jiao-Tong University School of Medicine, No. 280 Chongqing South Road, Shanghai 200025, P.R. China

E-mail: yanhua_candy@yahoo.com.cn

${ }^{*}$ Contributed equally

Key words: multiple myeloma, isobavachalcone, chloroquine, apoptosis, autophagy, protein kinase $\mathrm{C} \delta$ relatively specific for cancer cells, the combination of these two agents at non-toxic or sub-toxic concentrations represents an attractive novel regimen for myeloma treatment and warrants further investigation in preclinical and clinical studies.

\section{Introduction}

Multiple myeloma is an age-related malignant disease characterized by the clonal proliferation of B-cell lineage plasma cells resulting in the production of monoclonal proteins in serum and/ or urine, and leads to destructive bony lesions, defective hematopoiesis and renal failure $(1,2)$. Although increasing numbers of therapeutics are becoming available to treat this disease with the potential for significant symptom palliation, the induction of effective antibody responses, and prolongation of disease-free survival, available therapeutic approaches including peripheral blood stem cell transplantation (3) and newer agents, such as bortezomib, thalidomide and lenalidomide (4-6), have had only a modest impact on patient overall survival in several randomized trials. Multiple myeloma remains invariably incurable. Therefore, a more effective treatment is urgently required for this disease (7).

Natural products are fertile source for revealing novel lead molecules. In fact, $67 \%$ of anticancer drugs are natural products or natural product derivatives (8). Among them, chalcones represent an important group of natural compounds, which are absorbed in the daily diet and appear to be promising cancer chemopreventive agents (9). Isobavachalcone (IBC), a naturally occurring chalcone compound derived from the seeds of Psoralea corylifolia L., has long been used in traditional Chinese medicine as anthelmintic, antibacterial, aphrodisiac, astringent and antiplatelet agent (10-12). Recently, IBC has also been shown to have anticancer activity $(13,14)$. IBC has been shown to exert an inhibitory effect against skin tumor promotion in vivo in mouse skin carcinogenesis (15), and to induce apoptosis in neuroblastoma (16). More importantly, IBC has been shown to be less toxic to normal cells, indicating that IBC is a promising candidate for cancer therapy.

In this study, we assessed the anti-myeloma effect of IBC. We found that IBC induces apoptosis and autophagy in myeloma cells, in which autophagy plays a protective role. Moreover, we found that chloroquine (CQ), the only clinically 
used authophagy inhibitor, at its non-toxic concentration can markedly enhance IBC-induced cell death in myeloma cells but not in normal peripheral blood mononuclear cells (PBMCs). We further demonstrate that the mitochondrial pathway and the protelytic activation of protein kinase $\mathrm{C} \delta(\mathrm{PKC} \delta)$ contribute to this combined effect. To our knowledge, this is the first report to show that IBC has anti-myeloma activity. The combination treatment of CQ and IBC warrants further investigation in preclinical or clinical studies.

\section{Materials and methods}

Cell culture and reagents. $\mathrm{H} 929$ multiple myeloma cells were cultured in RPMI-1640 medium (Sigma-Aldrich, St. Louis, MO), supplemented with $10 \%$ fetal bovine serum (HyClone, Logan, UT). IBC was obtained from the Tauto Biotech Co., Ltd., Shanghai China, with $>98 \%$ purity as judged by HPLC analysis. IBC were dissolved in dimethyl sulfoxide (DMSO). CQ was purchased from Sigma. 3-Methyladenine (3-MA) was obtained from Sigma and dissolved in distilled water. Bafilomycin A was obtained from LC laboratories (Woburn, MA) and dissolved in DMSO as $1 \mathrm{mM}$ stock solution. Rottlerin (Biomol, Plymouth, PA) was prepared in DMSO as $2 \mathrm{mM}$ stock solution.

Isolation of PBMCs. Heparinized venous blood was obtained from two healthy human volunteers with their mutual consent. Mononuclear cells were isolated in a Ficoll-Hypaque (Pharmacia, Piscataway, NJ) density gradient using standard procedures which separated PBMCs from whole blood. The buffy coat containing PBMCs was removed carefully following centrifugation and washed twice in RPMI-1640 medium containing 10\% FBS. Cell viability determined by trypan blue exclusion was above $90 \%$. Cells $\left(1 \times 10^{5}\right)$ were incubated in $100 \mu \mathrm{l}$ medium in round-bottom 96-well plates and were cultured in the presence of CQ and/or IBC for $48 \mathrm{~h}$ and cell proliferation was determined by the Cell Counting kit-8 (CCK-8) assay as described previously (17).

Apoptosis assay. Apoptosis was measured by the Annexin V Fluos apoptosis detection kit (Roche Molecular Biochemicals, Mannheim, Germany) following the manufacturer's instructions. Annexin V-positive and PI-negative cells were considered to be in the early apoptotic phase and those having positive staining both for Annexin V and PI were deemed to undergo late apoptosis or necrosis.

Western blot analysis. The cells were washed with PBS and lysed with lysis buffer $(62.5 \mathrm{mM}$ Tris- $\mathrm{HCl}, \mathrm{pH} 6.8,100 \mathrm{mM}$ DTT, $2 \%$ SDS, $10 \%$ glycerol). Cell lysates were centrifuged at $20,000 \mathrm{x} \mathrm{g}$ for $10 \mathrm{~min}$ at $4^{\circ} \mathrm{C}$, and proteins in the supernatants were quantified. Protein extracts were equally loaded to an $8 \%$ to $15 \%$ SDS-polyacrylamide gel, electrophoresed, and transferred to nitrocellulose membrane (Amersham Bioscience, Buckinghamshire, UK). The blots were stained with $0.2 \%$ Ponceau $\mathrm{S}$ red to ensure equal protein loading. After blocking with 5\% non-fat milk in PBS for $1 \mathrm{~h}$ at room temperature, the membranes were probed with antibodies against cleaved caspase-3 and -9 (Cell Signaling Technology, Inc., Beverly, MA), Bcl-2, PKCס (Santa Cruz Biotechnology, Inc., Santa Cruz, CA), and poly (ADP-ribose) polymerase (PARP) (from BD Pharmingen ${ }^{\mathrm{TM}}$ ), LC3 (Sigma). The signals were detected by the chemiluminescence phototope-HRP kit (Cell Signaling Technology, Inc.) according to the manufacturer's instructions. Where necessary, the blots were stripped and reprobed with anti-actin antibody (Oncogene, Fremont, CA) as the internal control. All experiments were repeated three times with similar results.

RNA interference and transfection. The retrovirus vector for beclin-1 protein suppression by short hairpin RNA (shRNA) interference was generated as described previously (18). Briefly, retrovirus with two target shRNAs (B-sh2 and B-sh3) and nontarget control shRNA (NC)-containing plasmids were packaged in HEK293T cells by co-transfecting with pSIREN-RetroQ, pEQPAM (containing gag-pol, produced by Dr Lishan Su, UNC Chapel Hill, USA) and VSVG (Clontech; T-334350). After transfection for $48 \mathrm{~h}$, the viral supernatant was collected, filter-sterilized and added into H929 cells ( $2 \times 10^{5}$ cells/well) in 6 -well plates with medium containing $8 \mu \mathrm{g} / \mathrm{ml}$ of polybrene (TR-1003-G; Millipore) and $0.75 \mu \mathrm{g} / \mathrm{ml}$ of puromycin (540411; Calbiochem) for the selection of stably transfected cells after another $48 \mathrm{~h}$.

Mitochondrial membrane potential $(\Delta \Psi m)$ assessment. After washing twice with PBS, approximately $10^{6}$ cells were incubated $\left(37^{\circ} \mathrm{C}, 30 \mathrm{~min}\right)$ with $10 \mu \mathrm{g} / \mathrm{ml}$ rhodamine 123 (Rh123), a cationic lipophilic fluorochrome absorbed by the mitochondria in proportion to the $\Delta \Psi \mathrm{m}$. Then, $50 \mu \mathrm{g} / \mathrm{ml} \mathrm{PI}$, a membrane impermeable DNA-binding dye, was added to the cells. Fluorescence intensities were determined by flow cytometry (BD Biosciences). A total of 10,000 cells were analyzed in each sample. All data were collected, stored and analyzed by LYSIS II software (BD Biosciences).

Statistical analysis. The Student's t-test was used to evaluate the differences between two groups. A P-value $<0.05$ was considered to indicate a statistically significant difference.

\section{Results}

IBC inhibits the proliferation of myeloma H929 cells via apoptosis and autophagy induction. To examine the antitumor activity of IBC on myeloma cells, H929 cells were treated with IBC at $0,10,20$ and $40 \mu \mathrm{M}$ for two days, and the cell proliferation and viability were determined by trypan blue exclusion assay. As shown in Fig. 1, IBC inhibited the proliferation of H929 cells in a dose- and time-dependent manner (Fig. 1A), and approximately $15 \%$ cells lost viability upon treatment with IBC at $20 \mu \mathrm{M}$ (50\% inhibition) for two days (Fig. 1B). The characterization of IBC-induced cell death was further investigated. Following treatment with $10-40 \mu \mathrm{M}$ IBC for $48 \mathrm{~h}$, the 17 - and 19-kDa cleaved fragments of caspase-3, the 37-kDa cleaved caspase-9 and the $85-\mathrm{kDa}$ fragments of PARP appeared in a dose- and time-dependent manner, indicating the involvement of apoptosis (Fig. 1C). We also determined the expression level of LC-3, a reliable marker of autophagosome formation. Tracking the conversion of LC3-I to LC3-II is indicative of autophagic activity. Of note, IBC treatment also increased the expression of LC-3 II in a dose- and time-dependent manner (Fig. 1D). These data suggest that IBC induces both apoptosis and autophagy in H929 cells. 
A

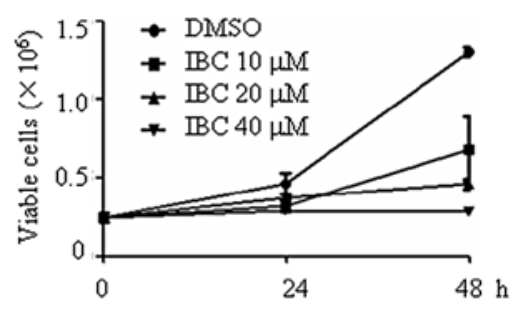

B

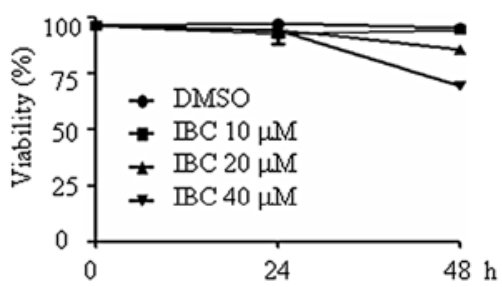

C

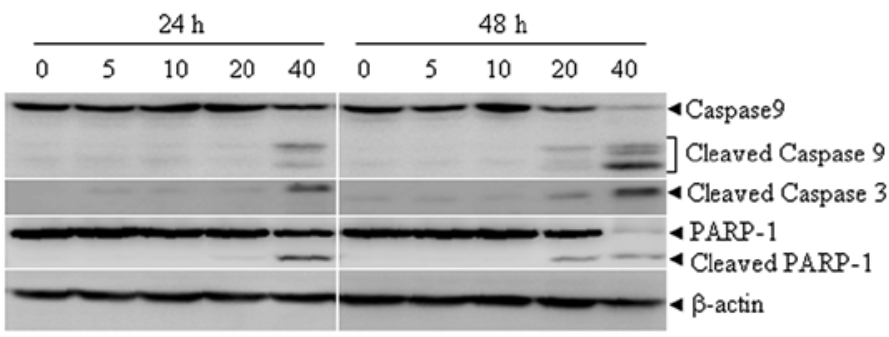

D

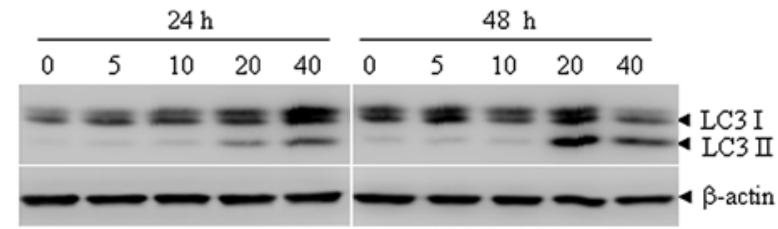

Figure 1. IBC inhibits the proliferation and induces apoptosis and autophagy of H929 multiple myeloma cells. (A and B) H929 cells were treated with various doses of IBC for the indicated times, and the (A) proliferation and (B) viability of cells were examined by trypan blue exclusion assay. Data expressed as the means \pm SD are representative of at least three independent experiments. (C and D) H929 cells were treated with various doses of IBC for 24 and 48 h, and the indicated protein concentrations were determined by western blot analysis.

A

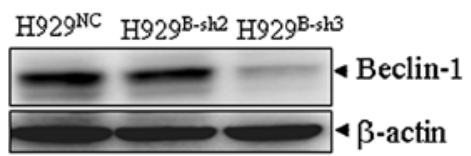

B

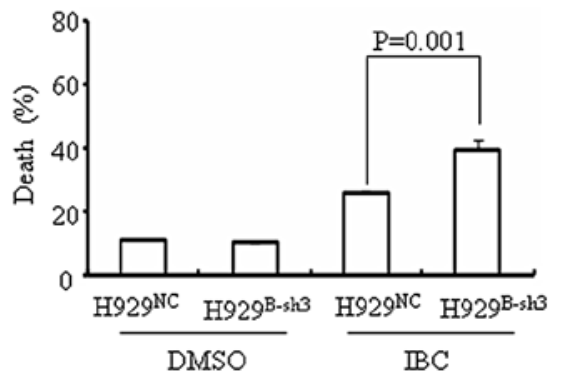

$\mathrm{C}$

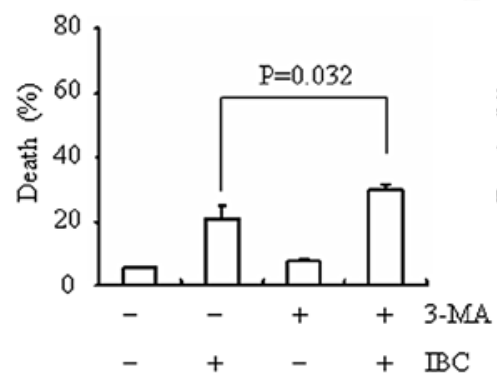

$\mathrm{D}$

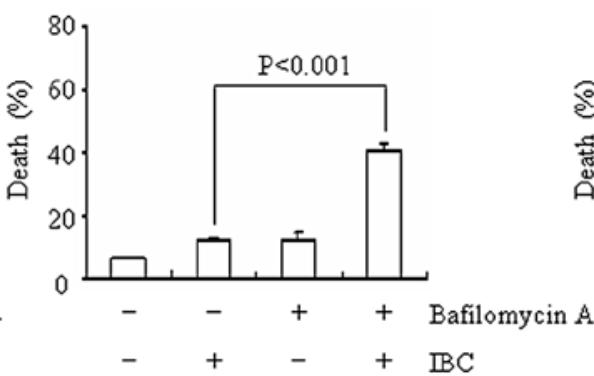

$\mathrm{E}$

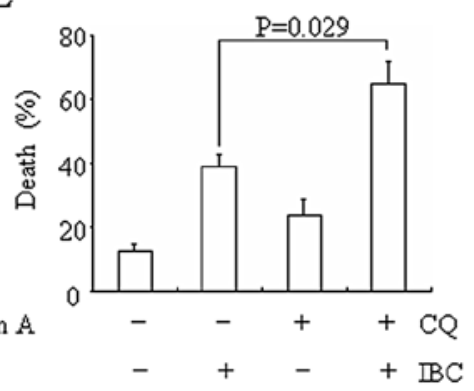

Figure 2. Knocking down of beclin-1 or co-treatment with the autophagy inhibitors, 3-MA, bafilomycin A, CQ, enhances IBC-induced cell death. (A) Beclin-1specific shRNA (H929 $\left.9^{\mathrm{B}-\mathrm{S} 2}, \mathrm{H} 929^{\mathrm{B}-\mathrm{S3}}\right)$ or non-specific shRNA $\left(\mathrm{H} 929^{\mathrm{NC}}\right)$ were stably transfected into H929 cells and the expression of beclin-1 was evaluated by western blot analysis. (B) H929 $9^{\mathrm{NC}}$ and H929 ${ }^{\mathrm{B}-\mathrm{S} 3}$ cells were treated with IBC for $48 \mathrm{~h}$, and the percentage of Annexin V-positive cells was examined by FACS. Data expressed as the means \pm SD are representative of at least three independent experiments. (C-E) H929 cells were treated with IBC in the presence or absence of 3-MA $(2 \mathrm{mM})$ or bafilomycin A $(5 \mathrm{nM})$ or CQ $(20 \mu \mathrm{M})$ for $48 \mathrm{~h}$, and the percentage of Annexin V-positive cells was evaluated by flow cytometry. Data expressed as the means \pm SD are representative of at least three independent experiments.

Knockdown of beclin-1 or co-treatment with autophagy inhibitors, 3-MA, bafilomycin A or CQ, enhances IBC-induced cell death. Autophagy can either be protective or induce programmed cell death. In most cases, autophagy acts as a survival mechanism and it has been shown that the inhibition of chemotherapy-induced autophagy frequently augments cell death (19). In order to examine the role of autophagy in IBC-induced cell death, two different approaches were used to inhibit autophagy. Firstly, beclin-1, an important autophagyrelated protein, was silenced by RNA interference in $\mathrm{H} 929$ cells. The target sequence, sh3, effectively downregulated the expression of beclin-1 (H929 ${ }^{\text {B-sh3 }}$ ) (Fig. 2A). Compared with the vector-transfected $\mathrm{H} 929$ cells $\left(\mathrm{H} 929^{\mathrm{NC}}\right)$, a significantly increased cell death was observed in the H929-sh3 cells upon treatment with IBC (Fig. 2B). Secondly, when used in combination with IBC, 3-MA, a well established autophagy 
A

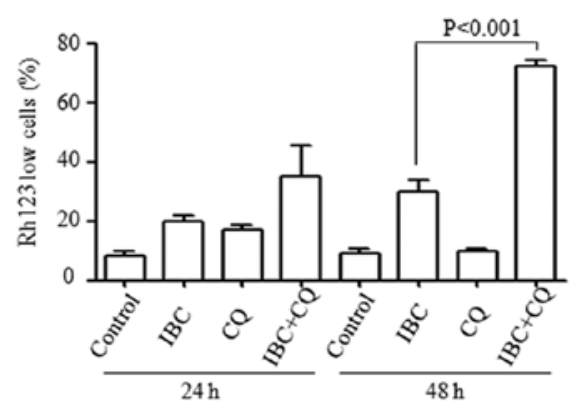

B

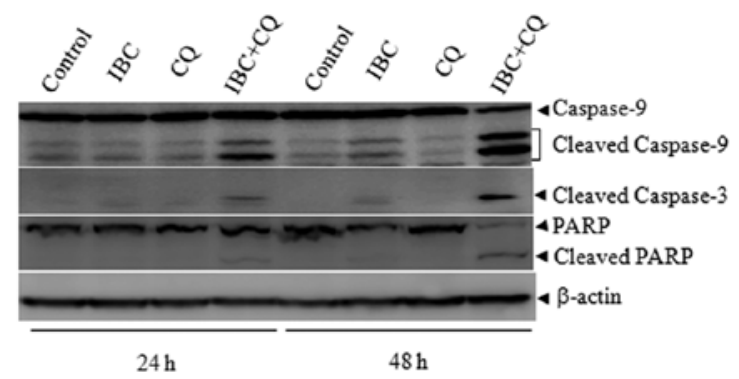

Figure 3. CQ plus IBC decreases the mitochondrial membrane potential and activates the apoptosis-related proteins. H929 cells were treated with IBC and $\mathrm{CQ}$ for $48 \mathrm{~h}$. (A) The mitochondrial membrane potential was determined by the Rh123/PI staining assay, and (B) the indicated protein concentrations were determined by western blot analysis, with $\beta$-actin as the loading control. Data expressed as the means \pm SD are representative of at least three independent experiments.

inhibitor which inhibits hVps34, a class III PI-3K (Fig. 2C) or bafilomycin $\mathrm{A}$, a specific vacuole-type $\mathrm{H}^{+}$-ATPase, which has been reported to disrupt the progression of autophagy at the later stage by inhibiting the fusion between autophagosomes and lysosomes (Fig. 2D), or CQ, which blocks lysosome and autophagosome fusion and lysosomal protein degradation, significantly enhanced IBC-induced cell death in H929 cells (Fig. 2E). Of note, $\mathrm{CQ}$ used at $20 \mu \mathrm{M}$ for $48 \mathrm{~h}$ was non-cytotoxic. Taken together, these data suggest that autophagy inhibition potentiates IBC-induced cell death.

Mitochondrial cell death pathway involved in $C Q$ plus $I B C$-induced cell death in H929 cells. CQ has been widely used as an autophagy inhibitor to enhance the efficacy of cancer therapeutic drugs and many clinical trials have used CQ for this purpose $(20,21)$. We then investigated the mechanism underlying the CQ plus IBC-enhanced cell death. It is known that the mitochondrial pathway plays a central role in determining cell survival or death in response to diverse stimuli and that the collapse of $\Delta \Psi \mathrm{m}$ is associated with mitochondrial dysfunction. We therefore assessed the effect of CQ plus IBC on mitochondrial $\Delta \Psi \mathrm{m}$ by Rh123/PI double staining assay. As depicted in Fig. 3A, compared with the single treatment of IBC, CQ plus IBC significantly increased the percentages of low Rh123-stained cells at $48 \mathrm{~h}(\mathrm{P}<0.001)$, suggesting that the dissipation of the $\Delta \Psi \mathrm{m}$ was associated with CQ plus IBC-induced cell death. Consistent with the collapse of $\Delta \Psi \mathrm{m}, \mathrm{CQ}$ plus IBC led to the obvious activation of caspase-9, an initiator caspase in the mitochondrial pathway. Accordingly, caspase-3 was
A

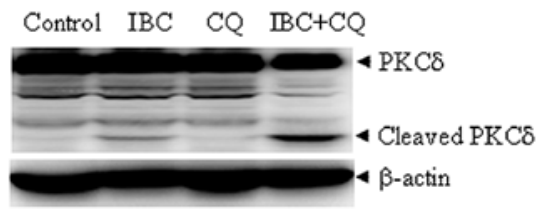

B

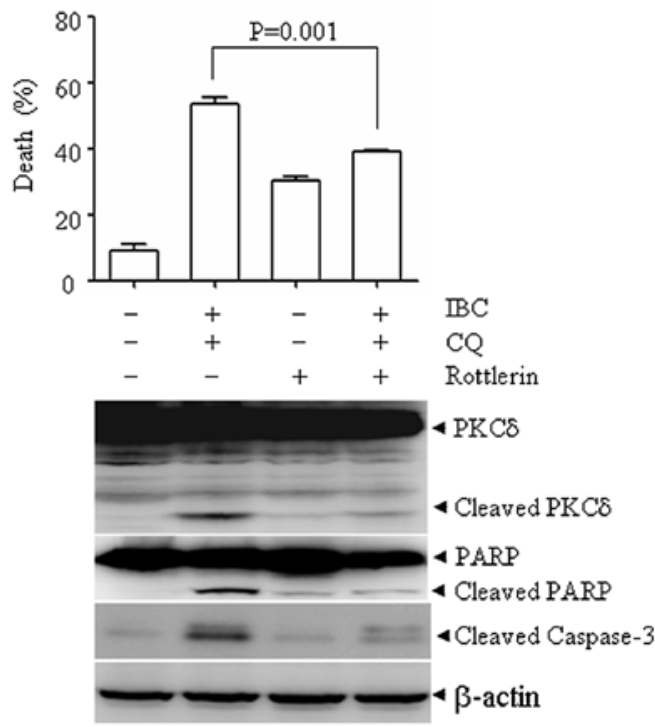

Figure 4. Proteolytic activation of PKC $\delta$ contributes to CQ plus IBC-induced apoptosis. (A) H929 cells were treated with CQ and/or IBC for $48 \mathrm{~h}$, and the expression of PKC $\delta$ was determined by western blot analysis. (B) H929 cells were treated with CQ plus IBC in the absence or presence of rottlerin for $30 \mathrm{~h}$, after which the percentage of Annexin V cells was determined by flow cytometry (upper panel), and the expression of the indicated proteins was examined by western blot analysis (lower panel). Data expressed as the means $\pm \mathrm{SD}$ are representative of at least three independent experiments.

also activated and PARP was cleaved into $85-\mathrm{kDa}$ fragments (Fig. 3B). These data suggest that the mitochondrial pathway contributes to CQ plus IBC-induced cell death.

Protelytic activation of $P K C \delta$ is involved in $C Q$ plus $I B C$-induced cell death. PKC $\delta$ activation has been shown to play a critical role in the mitochondrial cell death pathway and a previous report showed that CQ can activate PKC $(22)$. Thus, we investigated whether PKC $\mathrm{C}$ is also involved in the combined action of CQ plus IBC. Of note, CQ plus IBC led to the protelytic cleavage of PKCס into a 41-kDa catalytic fragment, which was associated with cell death (Fig. 4A). To evaluate the possible role of PKC $\delta$ in CQ plus IBC-induced cell death, we treated the H929 cells with CQ plus IBC in the presence or absence of rottlerin, a specific PKC $\delta$ inhibitor for $30 \mathrm{~h}$. As shown in Fig. 4B, rottlerin at $2 \mu \mathrm{M}$ significantly inhibited CQ plus IBC-induced cell death, as evidenced by the decrease in the number of Annexin V-positive cells (upper panel). Accordingly, the CQ plus IBC-induced proteolytic cleavage of $\mathrm{PKC} \delta$, the cleavage of caspase-3 and PARP were also inhibited (lower panel). These data indicate that PKC $\delta$ plays an important role in CQ plus IBC-induced cell death.

$C Q$ plus IBC exhibits low toxicity to normal PBMCs. To evaluate the possible effect of CQ plus IBC on normal cells, two samples of PBMCs (Fig. 5) were used. At the concentration 
A

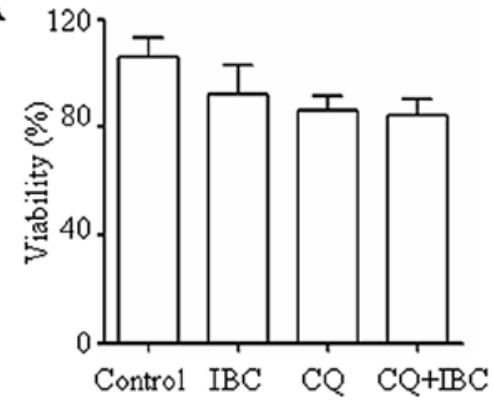

Sample 1
B

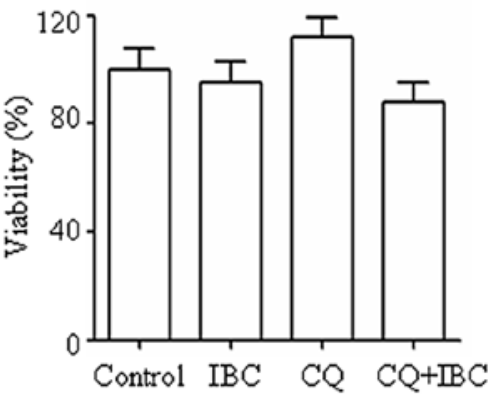

Sample 2

Figure 5. CQ plus IBC do not induce significant cell death in normal PBMCs. (A and B) Two PBMC samples were collected and treated with IBC and/or CQ for $48 \mathrm{~h}$, and the viability of the cells was evaluated using the CCK- 8 assay as described in Materials and methods. Data expressed as the means \pm SD are representative of at least three independent experiments.

used, CQ plus IBC did not significantly reduce cell viability in normal PBMCs. In combination with the results showing the cell death-enhancing effect of CQ plus IBC on H929 cells, these data indicate that myeloma cells are more sensitive than PBMCs to CQ plus IBC-induced cell death.

\section{Discussion}

Despite recent advances in the understanding of the disease pathogenesis and the introduction of velcade, thalidomide and other agents, multiple myeloma remains incurable. Therefore, the development of a novel therapeutic strategy to improve the outcome is urgently required. In this study, we demonstrate that IBC has anti-myeloma activity and that autophagy inhibition selectively augments the pro-apoptotic activity of IBC in myeloma cells but not in normal PBMCs cells. Furthermore, we demonstrate that the cell death-enhancing effect of $\mathrm{CQ}$ plus IBC is associated with the mitochondrial pathway and the protelytic activation of PKCס.

Autophagy is a highly conserved catabolic programme for degrading proteins and organelles. The role of autophagy in cell death is controversial. Autophagy can act as a survival or a cell death mechanism (23). Accumulating data have shown that many chemotherapy drugs can induce apoptosis accompanied by the activation of autophagy, which provides a protective mechanism for cancer cells. Therefore, combination treatment with autophagy inhibitors is an attracting strategy to enhance the efficacy of chemotherapy (24). As IBC-induced cell death involves both apoptosis and autophagy, we hypothesized that autophagy inhibition may enhance the cell killing effect of IBC. In support of this notion, the autophagy inhibition achieved by knocking down beclin-1, a specific autophagy-promoting protein, or by using the autophagy inhibitors, 3-MA, bafilomycin A and CQ, significantly enhanced IBC-induced cell death. These data suggest that the inhibition of autophagy in IBC-treated myeloma cells may switch the pro-survival effect of autophagy to apoptosis.

Mitochondria are the main regulators of apoptotic cell death. Anticancer drugs including IBC may damage the mitochondria by increasing the permeability of the outer mitochondrial membrane, which is associated with the collapse of $\Delta \Psi \mathrm{m}$ (25). The mitochondria have also been shown to be the central organelles integrating autophagy and apoptosis (26).
Based on these findings, we examined the role of mitochondria in CQ plus IBC-induced cell death. Compared with the single treatment of IBC or CQ, an obvious collapse of $\Delta \Psi \mathrm{m}$ was observed following CQ plus IBC treatment, which was paralleled with the increase in apoptosis. These data indicate that the inhibition of autophagy enhances the dysfunction of the mitochondria, which in turn augments the apoptotic cell death. We then investigated the possible mechanisms underlying mitochondrial dysfunction mediated by the co-treatment of CQ and IBC. Due to its important role in the regulation of the mitochondrial cell death pathway, $\mathrm{PKC} \delta$, a ubiquitously expressed member of the novel PKC family, was examined $(27,28)$. Indeed, paralleled with the loss of $\Delta \Psi \mathrm{m}, \mathrm{CQ}$ plus IBC caused the proteolytic activation of $\mathrm{PKC} \delta$ and rottlerin, an inhibitor of PKCס, significantly preventing CQ plus IBC-induced cell death, indicating the important contribution of PKC $\delta$ to cell death. On the contrary, $\mathrm{Ni}$ et al (29) reported that, PKC $\delta$ is commonly expressed in myeloma cells and plays an important role in plasma cell survival. This discrepancy may be explained by the fact that the PKC $\delta$ catalytic fragment has pro-apoptotic activity, whereas the holoenzyme induces the opposite effect. We do not rule out other possibilities. For example, the CQ-induced autophagy inhibition may eventually disrupt the lysosome and lead to the release of lysosome proteases into the cytosol, which would eventually lead to the dysfunction of the mitochondria.

Combination therapy is an important strategy to improve the outcome of multiple myeloma. Our results showed that CQ plus IBC may be a valuable regimen for the treatment of multiple myeloma. Firstly, IBC has potential value in clinical applications. IBC displayed a satisfactory selectivity between cancer cells and normal cells. The safety of IBC is also indirectly reflected by the fact that $\mathrm{IBC}$ is an active ingredient from the seeds of Psoralea corylifolia L. and the crude drug has been used in certain Chinese traditional medicines, such as 'Qing E Wan' for the treatment of senile osteoporosis and arthralgia. Secondly, CQ is a well known 4-aminoquinoline class of drug that is widely used as an anti-malaria (30), antiinflammatory and antiviral agent (31). Recently, CQ has been studied for its potential in the enhancement of radiation therapy, chemotherapy, and combination therapy for cancer $(21,32)$. The published data suggest that the combined modalities of CQ with other therapeutics are very promising for increasing 
therapeutic efficacy and decreasing undesirable side-effects. For example, CQ has been shown to dramatically increase the killing power of Gleevec and SAHA against chronic myeloma leukemia cells $(33,34)$. More importantly, our results showed that CQ plus IBC at a relatively low concentration caused significant cell death in myeloma cells but not in normal PBMCs, indicating that this combination is more selective to cancer cells.

In conclusion, we demonstrate for the first time IBC has anti-myeloma activity, and that CQ can markedly enhance IBC-induced cell death in myeloma cells, in which the mitochondrial pathway and $\mathrm{PKC} \delta$ activation play important roles. As CQ and IBC have been shown to be relatively specific to cancer cells, the combination treatment of both agents at nontoxic or sub-toxic concentration warrants further investigation in preclinical and clinical studies.

\section{Acknowledgements}

The present study was supported in part by grants from the National Basic Research Program of China (973 Program) (no. 2010CB912104), the National Natural Science Foundation of China (nos. 81172322, 81070433, 91013008), the Science and Technology Committee of Shanghai (no. 11JC1406500), the SMC Program of Shanghai Jiao Tong University.

\section{References}

1. Mahindra A, Hideshima T and Anderson KC: Multiple myeloma: biology of the disease. Blood Rev 24 (Suppl 1): S5-S11, 2010.

2. Dimopoulos MA and Terpos E: Multiple myeloma. Ann Oncol 21 (Suppl 7): S143-S150, 2010.

3. Giralt S: Stem cell transplantation for multiple myeloma: current and future status (Review). Hematology Am Soc Hematol Educ Program 2011: 191-196, 2011.

4. Chanan-Khan AA, San Miguel J, Jagannath S, Ludwig H and Dimopoulos AM: Novel therapeutic agents for the management of multiple myeloma patients with renal impairment. Clin Cancer Res 18: 2145-2163, 2012.

5. Mariz JM and Esteves GV: Review of second-line therapy for multiple myeloma: focus on lenalidomide. Curr Opin Oncol 24 (Suppl 2): S3-S11, 2012.

6. Kropff M, Baylon HG, Hillengass J, et al: Thalidomide versus dexamethasone for the treatment of relapsed and/or refractory multiple myeloma: results from OPTIMUM, a randomized trial Haematologica 97: 784-791, 2011.

7. Mahindra A, Laubach J, Raje N, Munshi N, Richardson PG and Anderson K: Latest advances and current challenges in the treatment of multiple myeloma. Nat Rev Clin Oncol 9: 135-143, 2012.

8. Balunas MJ and Kinghorn AD: Drug discovery from medicinal plants. Life Sci 78: 431-441, 2005.

9. Orlikova B, Tasdemir D, Golais F, Dicato M and Diederich M: Dietary chalcones with chemopreventive and chemotherapeutic potential. Genes Nutr 6: 125-147, 2011.

10. Haraguchi H, Inoue J, Tamura Y and Mizutani K: Antioxidative components of Psoralea corylifolia (Leguminosae). Phytother Res 16: 539-544, 2002.

11. Jantan I, Mohd Yasin YH, Jamil S, Sirat H and Basar N: Effect of prenylated flavonoids and chalcones isolated from Artocarpus species on platelet aggregation in human whole blood. J Nat Med 64: 365-369, 2010.
12. Kuete V, Ngameni B, Tangmouo JG, et al: Efflux pumps are involved in the defense of Gram-negative bacteria against the natural products isobavachalcone and diospyrone. Antimicrob Agents Chemother 54: 1749-1752, 2010.

13. Jing H, Zhou X, Dong X, et al: Abrogation of Akt signaling by Isobavachalcone contributes to its anti-proliferative effects towards human cancer cells. Cancer Lett 294: 167-177, 2010.

14. Szliszka E, Czuba ZP, Mazur B, Sedek L, Paradysz A and Krol W: Chalcones enhance TRAIL-induced apoptosis in prostate cancer cells. Int J Mol Sci 11: 1-13, 2009.

15. Ohno O, Watabe T, Nakamura K, et al: Inhibitory effects of bakuchiol, bavachin, and isobavachalcone isolated from Piper longum on melanin production in B16 mouse melanoma cells. Biosci Biotechnol Biochem 74: 1504-1506, 2010.

16. Nishimura R, Tabata K, Arakawa M, et al: Isobavachalcone, a chalcone constituent of Angelica keiskei, induces apoptosis in neuroblastoma. Biol Pharm Bull 30: 1878-1883, 2007.

17. Zhao Y, Pu JX, Huang SX, et al: ent-Kaurane diterpenoids from Isodon pharicus. J Nat Prod 72: 988-993, 2009.

18. Huang Y, Hou JK, Chen TT, et al: PML-RARalpha enhances constitutive autophagic activity through inhibiting the Akt/ mTOR pathway. Autophagy 7: 1132-1144, 2011.

19. Shen S, Kepp O and Kroemer G: The end of autophagic cell death? Autophagy 8: 1-3, 2012.

20. Han W, Sun J, Feng L, et al: Autophagy inhibition enhances daunorubicin-induced apoptosis in K562 cells. PLoS One 6: e28491, 2011.

21. Solomon VR and Lee H: Chloroquine and its analogs: a new promise of an old drug for effective and safe cancer therapies. Eur J Pharmacol 625: 220-233, 2009.

22. Chen TH, Chang PC, Chang MC, Lin YF and Lee HM: Chloroquine induces the expression of inducible nitric oxide synthase in C6 glioma cells. Pharmacol Res 51: 329-336, 2005.

23. Denton D, Nicolson S and Kumar S: Cell death by autophagy: facts and apparent artefacts. Cell Death Differ 19: 87-95, 2012.

24. Mancias JD and Kimmelman AC: Targeting autophagy addiction in cancer. Oncotarget 2: 1302-1306, 2011.

25. Kroemer G, Galluzzi L and Brenner C: Mitochondrial membrane permeabilization in cell death. Physiol Rev 87: 99-163, 2007.

26. Gozuacik D and Kimchi A: Autophagy as a cell death and tumor suppressor mechanism. Oncogene 23: 2891-2906, 2004.

27. Steinberg SF: Distinctive activation mechanisms and functions for protein kinase Cdelta. Biochem J 384: 449-459, 2004.

28. Jackson DN and Foster DA: The enigmatic protein kinase Cdelta: complex roles in cell proliferation and survival. FASEB J 18: 627-636, 2004.

29. Ni H, Ergin M, Tibudan SS, Denning MF, Izban KF and Alkan S: Protein kinase C-delta is commonly expressed in multiple myeloma cells and its downregulation by rottlerin causes apoptosis. Br J Haematol 121: 849-856, 2003.

30. Rathore D, McCutchan TF, Sullivan M and Kumar S: Antimalarial drugs: current status and new developments. Expert Opin Investig Drugs 14: 871-883, 2005.

31. Cooper RG and Magwere T: Chloroquine: novel uses \& manifestations. Indian J Med Res 127: 305-316, 2008.

32. Maycotte P, Aryal S, Cummings CT, Thorburn J, Morgan MJ and Thorburn A: Chloroquine sensitizes breast cancer cells to chemotherapy independent of autophagy. Autophagy 8: 200-212, 2012.

33. Bellodi C, Lidonnici MR, Hamilton A, et al: Targeting autophagy potentiates tyrosine kinase inhibitor-induced cell death in Philadelphia chromosome-positive cells, including primary CML stem cells. J Clin Invest 119: 1109-1123, 2009.

34. Carew JS, Nawrocki ST, Kahue CN, et al: Targeting autophagy augments the anticancer activity of the histone deacetylase inhibitor SAHA to overcome Bcr-Abl-mediated drug resistance. Blood 110: 313-322, 2007. 